\title{
Recent results on (anti)nucleus and (anti)hyperon production in nucleus-nucleus collisions at CERN SPS energies
}

\section{Georgy Melkumov* for the NA49 Collaboration}

Joint Institute for Nuclear Research, Dubna

E-mail: Georgui.Melkoumov@cern.ch

\section{The NA49 Collaboration:}

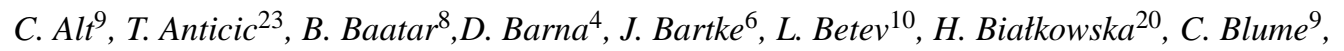
B. Boimska ${ }^{20}$, M. Botje ${ }^{1}$, J. Bracinik ${ }^{3}$, R. Bramm ${ }^{9}$, P. Bunčić10 ${ }^{10}$ V. Cerny ${ }^{3}$, P. Christakoglou ${ }^{2}$, P. Chung ${ }^{19}$, O. Chvala ${ }^{14}$, J.G. Cramer ${ }^{16}$, P. Csató $^{4}$, P. Dinkelaker ${ }^{9}$, V. Eckardt ${ }^{13}$, D. Flierl ${ }^{9}$, Z. Fodor ${ }^{4}$, P. Foka ${ }^{7}$, V. Friese ${ }^{7}$, J. Gál ${ }^{4}$, M. Gaździcki ${ }^{9,11}$, V. Genchev ${ }^{18}$, G. Georgopoulos ${ }^{2}$, E. Gtadysz ${ }^{6}$, K. Grebieszkow ${ }^{22}$, S. Hegyi ${ }^{4}$, C. Höhne ${ }^{7}, K_{\text {Kadija }}^{23}$, A. Karev $^{13}$, D. Kikola ${ }^{22}$, M. Kliemant ${ }^{9}$, S. Kniege ${ }^{9}$, V.I. Kolesnikov ${ }^{8}$, E. Kornas $^{6}$, R. Korus ${ }^{11}$, M. Kowalski ${ }^{6}$, I. Kraus ${ }^{7}$, M. Kreps ${ }^{3}$, A. Laszlo ${ }^{4}$, R. Lacey ${ }^{19}$, M. van Leeuwen ${ }^{1}$, P. Lévai ${ }^{4}$, L. Litov ${ }^{17}$, B. Lungwitz ${ }^{9}$, M. Makariev ${ }^{17}$, A.I. Malakhov ${ }^{8}$, M. Mateev ${ }^{17}$, G.L.Melkumov ${ }^{8}$, A. Mischke ${ }^{1}$, M. Mitrovski ${ }^{9}$, J. Molnár ${ }^{4}$, St. Mrówczyński ${ }^{11}$, V. Nicolic ${ }^{23}$, G. Pálla ${ }^{4}$, A.D. Panagiotou ${ }^{2}$, D. Panayotov ${ }^{17}$, A. Petridis ${ }^{2, \dagger}$,W. Peryt ${ }^{22}$, M. Pikna ${ }^{3}$, J. Pluta ${ }^{22}$, D. Prindle ${ }^{16}$, F. Pühlhofer ${ }^{12}$, R. Renfordt ${ }^{9}$, C. Roland ${ }^{5}$, G. Roland ${ }^{5}$, M. Rybczyński ${ }^{11}$, A. Rybicki ${ }^{6}$, A. Sandoval ${ }^{7}$, N. Schmitz ${ }^{13}$, T. Schuster ${ }^{9}$, P. Seyboth ${ }^{13}$, F. Siklér ${ }^{4}$, B. Sitar ${ }^{3}$, E. Skrzypczak ${ }^{21}$, M. Slodkowski ${ }^{22}$, G. Stefanek ${ }^{11}$, R. Stock ${ }^{9}$, C. Strabel ${ }^{9}$, H. Ströbele ${ }^{9}$, T. Susa ${ }^{23}$, I. Szentpétery ${ }^{4}$, J. Sziklai $^{4}$, M. Szuba $^{22}$, P. Szymanski $^{10,20}$, V. Trubnikov ${ }^{20}$, D. Varga ${ }^{4,10}$, M. Vassiliou ${ }^{2}$, G.I. Veres ${ }^{4,5}$, G. Vesztergombi $^{4}$, D. Vranic $^{7}$, A. Wetzler ${ }^{9}$, Z. Włodarczyk ${ }^{11}$, A. Wojtaszek ${ }^{11}$, I.K. Yoo ${ }^{15}$, J. Zimányi ${ }^{4, \dagger}$ 
${ }^{1}$ NIKHEF, Amsterdam, Netherlands.

${ }^{2}$ Department of Physics, University of Athens, Athens, Greece.

${ }^{3}$ Comenius University, Bratislava, Slovakia.

${ }^{4}$ KFKI Research Institute for Particle and Nuclear Physics, Budapest, Hungary.

${ }^{5}$ MIT, Cambridge, USA.

${ }^{6}$ Henryk Niewodniczanski Institute of Nuclear Physics, Polish Academy of Sciences, Cracow, Poland.

${ }^{7}$ Gesellschaft für Schwerionenforschung (GSI), Darmstadt, Germany.

${ }^{8}$ Joint Institute for Nuclear Research, Dubna, Russia.

${ }^{9}$ Fachbereich Physik der Universität, Frankfurt, Germany.

${ }^{10}$ CERN, Geneva, Switzerland.

${ }^{11}$ Institute of Physics Świętokrzyska Academy, Kielce, Poland.

${ }^{12}$ Fachbereich Physik der Universität, Marburg, Germany.

${ }^{13}$ Max-Planck-Institut für Physik, Munich, Germany.

${ }^{14}$ Charles University, Faculty of Mathematics and Physics, Institute of Particle and Nuclear Physics, Prague, Czech Republic.

${ }^{15}$ Department of Physics, Pusan National University, Pusan, Republic of Korea.

${ }^{16}$ Nuclear Physics Laboratory, University of Washington, Seattle, WA, USA.

${ }^{17}$ Atomic Physics Department, Sofia University St. Kliment Ohridski, Sofia, Bulgaria.

${ }^{18}$ Institute for Nuclear Research and Nuclear Energy, Sofia, Bulgaria.

${ }^{19}$ Department of Chemistry, Stony Brook Univ. (SUNYSB), Stony Brook, USA.

${ }^{20}$ Institute for Nuclear Studies, Warsaw, Poland.

${ }^{21}$ Institute for Experimental Physics, University of Warsaw, Warsaw, Poland.

${ }^{22}$ Faculty of Physics, Warsaw University of Technology, Warsaw, Poland.

${ }^{23}$ Rudjer Boskovic Institute, Zagreb, Croatia.

${ }^{\dagger}$ deceased

The NA49 experiment has collected comprehensive data on particle production in nucleus-nucleus collisions over the whole SPS beam energies range, the critical energy domain where the expected phase transition to a deconfined phase is expected to occur. The latest results from $\mathrm{Pb}+\mathrm{Pb}$ collisions between $20 \mathrm{~A} \mathrm{GeV}$ and $158 \mathrm{~A} \mathrm{GeV}$ on baryon stopping and light nuclei production as well as those for strange hyperons are presented.

The measured data on $p, \bar{p}, \Lambda, \bar{\Lambda}, \Xi^{-}$and $\bar{\Xi}^{+}$production were used to evaluate the rapidity distributions of net-baryons at SPS energies and to compare with the results from the AGS and the RHIC for central $\mathrm{Pb}+\mathrm{Pb}(\mathrm{Au}+\mathrm{Au})$ collisions.

The dependence of the yield ratios and the inverse slope parameter of the $m_{t}$ spectra on the collision energy and centrality, and the mass number of the produced nuclei ${ }^{3} \mathrm{He}, t, d$ and $\bar{d}$ are discussed within coalescence and statistical approaches. Analysis of the total multiplicity exhibits remarkable agreement between the measured yield for ${ }^{3} \mathrm{He}$ and those predicted by the statistical hadronization model.

In addition, new results on $\Lambda$ and $\bar{\Lambda}$ as well as $\Xi^{-}$production in minimum bias $\mathrm{Pb}+\mathrm{Pb}$ reactions at $40 \mathrm{~A} \mathrm{GeV}$ and $158 \mathrm{~A} \mathrm{GeV}$ and central $\mathrm{C}+\mathrm{C}, \mathrm{Si}+\mathrm{Si}$ and $\mathrm{Pb}+\mathrm{Pb}$ collisions are presented. The system size dependence of the yields of these hyperons was analysed to determine the evolution of strangeness enhancement relative to elementary $p+p$ collisions.

Critical Point and Onset of Deconfinement 4th International Workshop

July 9-132007

GSI Darmstadt,Germany 


\section{Introduction}

The NA49 experiment studied high energy and density matter produced in nucleus-nucleus collisions at the CERN SPS to search for evidence of quark-gluon deconfinement in the early stage of the reactions predicted by lattice QCD. In the first measurements NA49 demonstrated that in central $\mathrm{Pb}+\mathrm{Pb}$ collisions top SPS energy the initial energy density well exceeds the critical value of about $1 \mathrm{GeV} / \mathrm{fm}^{3}$. The results of the energy scan measurements between $20 \mathrm{~A} \mathrm{GeV}$ and $158 \mathrm{~A}$ $\mathrm{GeV}$ beam energies suggest that deconfinement indeed starts in the lower SPS energy range $[1,2]$. Among a primary features, the SPS experiments found that the relativistic heavy ion reactions produce an explosively expanding fireball with strong transverse and longitudinal flow. Another characteristics is that the ratios of yields of produced particles are consistent with statistical equilibration.

In this report a new results related to the formation of hot and dense nuclear matter in $A-A$ collisions and its dynamics - energy dependence of baryon stopping and light nucleus production, and possible evidence of deconfinement - size dependence of strangeness enhancement will be presented.

\section{Experiment}

The experiment was carried out with the NA49 large acceptance hadron detector [3] employing a system of time projection chambers (TPCs) for efficient tracking in the forward hemisphere of the reactions, precise momentum reconstruction in the magnetic field and particle identification using the energy loss $d E / d x$ in the TPC gas. Two time of flight (TOF) walls of 900 scintillator pixels each situated symmetrically behined TPCs augment particle identification near midrapidity. A zero degree calorimeter ZDC is used for centrality selection of events in nucleus -nucleus collisions at triggering as well as in the off-line analysis.

Raw charged particle yields were obtained by unfolding the distributions of $d E / d x$ and from TOF measurements in small bins of momentum $p$ and transverse momentum $p_{t}[4,5]$. Strange particles $\left(K_{S}^{0}, \Lambda, \Xi, \Omega\right)$ are detected via decay topology and invariant mass measurement $[6,7]$. A various type of corrections have been applied to the data, such as corrections for geometrical acceptance, reconstruction efficiency, in-flight particle decay and feeddown from weak decays.

\section{Baryon stopping}

It is important in relativistic heavy ion experiments to understand nuclear stopping in details since it is a requisite for the formation of hot and dense nuclear matter. Stopping is a necessary ingradient in our overall understanding of the reaction and the expectations to form and study the properties of QGP.

New results on rapidity spectra of protons and antiprotons in central $\mathrm{Pb}+\mathrm{Pb}$ reactions at $20 \mathrm{~A}$ - 80A GeV shown in Fig. 1 in combination with previously published results $[8,5]$ allow to study the energy evolution of stopping. Based on the measured rapidity spectra for $p,{ }^{-} p \Lambda, \bar{\Lambda}, \Xi^{-}$and

\footnotetext{
* Speaker.
} 

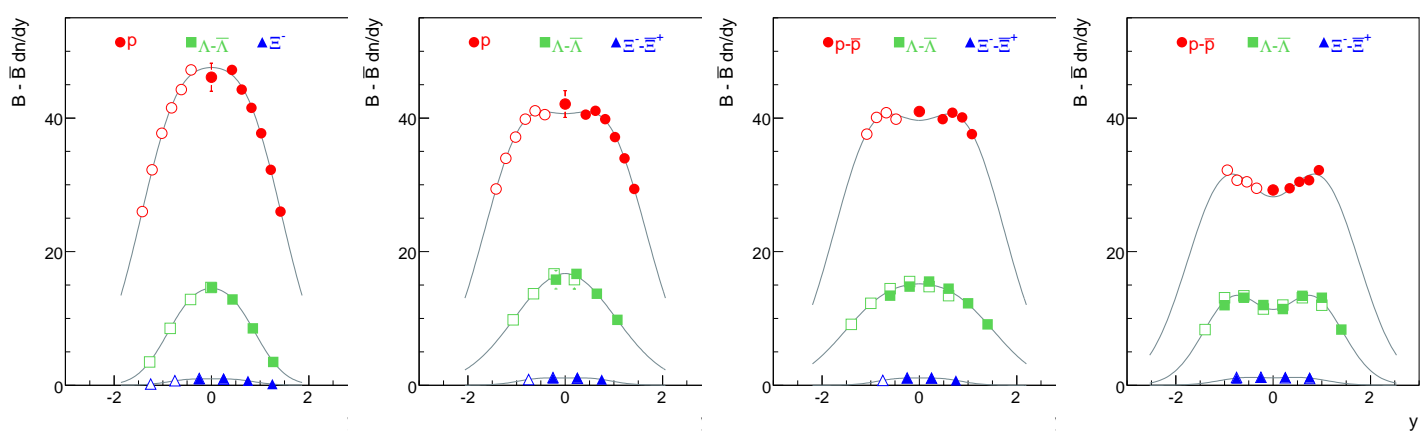

Figure 1: Rapidity distributions of net baryons $p, \Lambda$ and $\Xi$ measured in the most $7 \%$ cemtral $\mathrm{Pb}+\mathrm{Pb}$ collisions at CERN SPS energies (left to right) $20 A, 30 A, 40 A, 80 A \mathrm{GeV}$. Open data points were obtained by reflection at midrapidity.
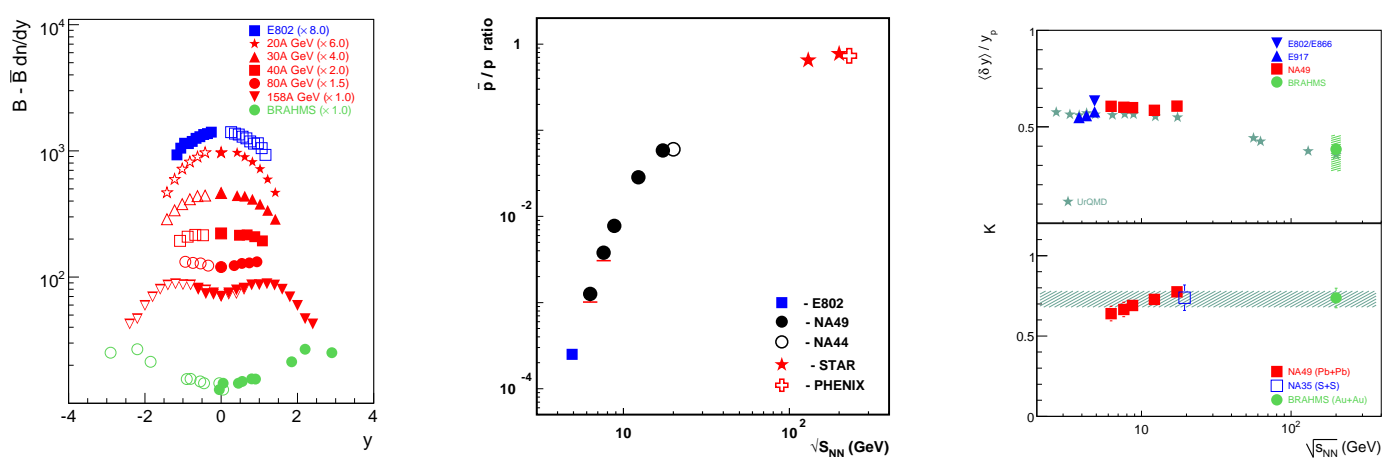

Figure 2: (left) Net baryon rapidity distributions at AGS[9], SPS[8] and RHIC[10] energies in central $\mathrm{Pb}+\mathrm{Pb}$ or $\mathrm{Au}+\mathrm{Au}$ collisions. Open data points were obtained by reflection. (center) $\bar{p} / p$ ratio at midrapidity as a function of $\sqrt{s_{N N}}$ in central $\mathrm{Pb}+\mathrm{Pb}(\mathrm{Au}+\mathrm{Au})$ collisions reviwed in [5]. (right top) Relative rapidity shifts of projectile nucleons extracted from net baryon distributions compared to results (stars) from the UrQMD model calculations [11]. (right bottom) Dependence of the inelasticity factor $K$ on $\sqrt{s_{N N}}$.

$\bar{\Xi}^{+}$(Fig. 1), all corrected for feed down from weak decays, the net-baryon distributions $\mathrm{d} N_{(\mathrm{B}-\overline{\mathrm{B}})} / \mathrm{d} y$ are constructed:

$$
B-\bar{B}=S_{n} \cdot\left(p-{ }^{-} p\right)+\mathcal{S}^{ \pm} \cdot(\Lambda-\bar{\Lambda})+S_{\Xi^{0}} \cdot\left(\Xi^{-}-\bar{\Xi}^{+}\right) .
$$

The contribution of unmeasured baryons $\left(\mathrm{n}, \Sigma^{ \pm}, \Xi^{0}\right.$ ) was estimated using a scaling factors $S_{x}$ obtained from statistical hadron gas model fits [12]. The results are plotted in Fig. 2 (left). A clear evolution is seen from a peak to a dip structure in the SPS energy range.

The averaged rapidity shift of projectile nucleons $\langle\delta y\rangle$, which is commonly used to quantify stopping in $A-A$ collisins, can be derived from:

$$
\langle\delta y\rangle=y_{\text {proj }}-\frac{2}{N_{\text {part }}} \int_{0}^{y_{\text {proj }}} y \frac{d N_{B-\bar{B}}}{d y} d y,
$$

where $y_{\text {proj }}$ is the projectile rapidity and $N_{\text {part }}$ is the number of participating nucleons. As seen from Fig. 2 (upper right), $\langle\delta y\rangle$ is approximately 0.6 at AGS and SPS and then slowly decreases in the 
RHIC energy range. This behaviour is well reproduced by the UrQMD model calculations [11].

Another measure promptly related to the stopping power is the ${ }^{-} p / p$ ratio and its dependence on the energy and centrality of the interactions. Fig. 2 (center) displays the midrapidity ${ }^{-} p / p$ ratio for central collisions as a function of energy at the AGS, SPS and RHIC early reviewed in [5]. The ratio rises steeply within the SPS energy range by nearly two orders of magnitude. The figure illustrates how the collisions evolve from producing a net baryon-rich system at the AGS through the SPS energy range to an almost net baryon-free midrapidity region at the RHIC.

The partial stopping of incident nucleons observed in $A-A$ collisions provides the energy for particle production, which can be estimated in terms of the inelasticity. Using $\mathrm{d} N_{(\mathrm{B}-\overline{\mathrm{B}})} / \mathrm{d} y$ and the measured $\left\langle m_{\mathrm{t}}\right\rangle$ the inelastic energy per net-baryon:

$$
E_{\text {inel }}=\frac{\sqrt{s_{\mathrm{NN}}}}{2}-\frac{1}{N_{(\mathrm{B}-\overline{\mathrm{B}})}} \int_{-y_{p r o j}}^{y_{p r o j}}\left\langle m_{\mathrm{t}}\right\rangle \frac{\mathrm{d} N_{(\mathrm{B}-\overline{\mathrm{B}})}}{\mathrm{d} y} \cosh y \mathrm{~d} y
$$

and the inelasticity factor $K=2 E_{\text {inel }} /\left(\sqrt{s_{\mathrm{NN}}}-2 m_{\mathrm{p}}\right)$ can be calculated. Fig. 2 (lower right) indicates that $K$ is approximatly energy independent and the average energy loss of projectiles amounts to about $70-80 \%$ at all energies.

\section{Production of light nuclei}

There is a notion that abandances of light nuclei probe the latest stage of the evolution of a systems created in relativistic heavy ion collision. After the produced system has cooled and expanded the nucleons in close proximity and moving with small relative momenta coalesce to form nuclei. Thus light nuclei production enables the study of several topics, including the mechanism of composite particle production, freeze-out temperature, size of the interaction region and enrtopy of the system. Production of light nuclei has traditionally been interpreted in terms of coalescence models $[13,14]$.

Recently NA49 has measured the energy dependence of deuteron snd tritons production at midrapidity, and ${ }^{3} \mathrm{He}$ nuclei in a wide rapidity range. A rare process of the antideuteron production at midrapidity in central $\mathrm{Pb}+\mathrm{Pb}$ collisions at $158 \mathrm{~A} \mathrm{GeV}$ beam energy was also studied. It is of significant interest because the initial colliding system contains no antibaryons therefore their yields and spectra are determined solely by the post collision dynamics. It is also reasonable in the antideuteron analysis to assume that the ratio of $\bar{d}$ to ${ }^{-} p$ prior to coalescence is close to 1 , whereas for deuterons the coalessence probability will depend upon the beam and target nuclei, as well as other details of the reaction. Additionally, the produced antideuterons have no contribution from spectator fragments.

In such investigations the identification of the produced nuclei is of primary importance. The Fig. 3 (left) demonstrates the clean identification of $d, t$ and ${ }^{3} \mathrm{He}$ from measurements of the energy loss $d E / d x$ in the TPC gas and mass squared $M^{2}$ computed from momentum and time of flight measurements in TOF detector (see [15] for more details). As shown in Fig. 3 (center), for doubly charged ${ }^{3} \mathrm{He}$ nuclei the $\mathrm{dE} / \mathrm{dx}$ measerement was sufficient to uniquely and cleanly determine its identity thus providing a large coverage in rapidity for measuring ${ }^{3} \mathrm{He}$ in TPCs. For $\bar{d}$ a strong $d E / d x$ and TOF cuts were required to provide a reliable particle identification. The $M^{2}$ distribution 

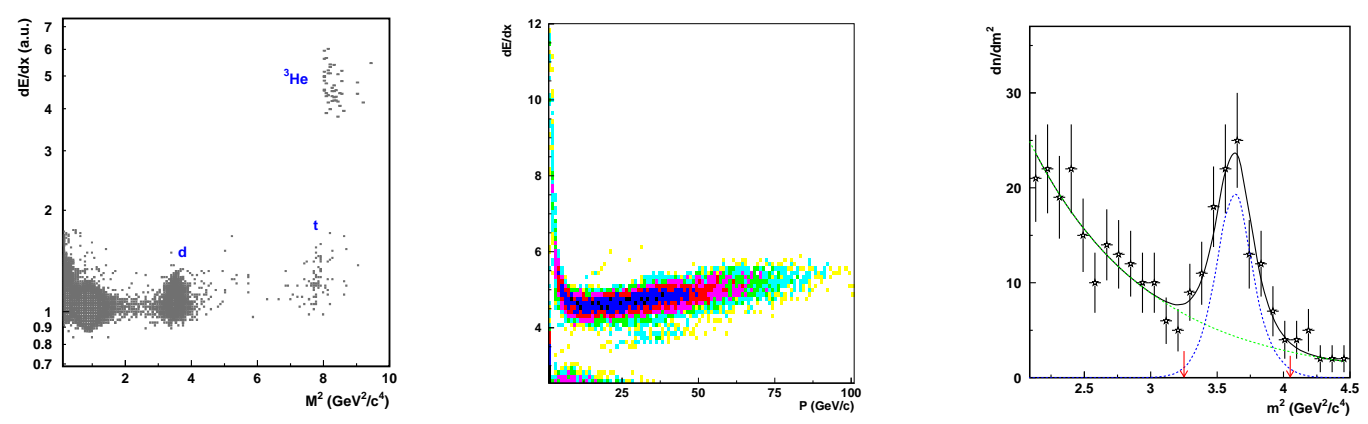

Figure 3: (left) Energy loss $d E / d x$ in TPC versus mass squared $M^{2}$ from measurements in TOF for ${ }^{3} H e, t$, and $d$ produced in central $\mathrm{Pb}+\mathrm{Pb}$ collisions. (center) Momentum dependence of the ${ }^{3} \mathrm{He}$ energy loss $d E / d x$. (right) Mass squared distribution for $\bar{d}$ after $d E / d x$ and TOF identication cuts.

after all identification cuts is shown on Fig. 3 (right). Antideuterons are clearly visible at about $M^{2} \approx 3.5 \mathrm{GeV}^{2} / c^{4}$.

New results on triton and ${ }^{3} \mathrm{He}$ production in central $\mathrm{Pb}+\mathrm{Pb}$ collisions at $20 \mathrm{~A}-80 \mathrm{~A} \mathrm{GeV}$ are shown in Fig. 4. The left and central panels display the invariant yields of $t$ and ${ }^{3} \mathrm{He}$ at midrapidity as a function of the trasverse mass $m_{t}$ for all four beam energies. Rapidity distributions $d n / d y$, summarised in the right panel of Fig. 4, were obtained by integration of the $m_{t}$ distributions in small bins of rapidity. One sees from the figure that the $d n / d y$ distributions have a concave shape at all investigated energies. This is in constrast to the case of protons, where the shape of rapidity distributions is rather convex and strongly energy dependent (see Fig. 1). The rapidity density distributions for light nuclei were studied in $[16,17,18]$ using the RQMD model in combination with a coalescence model applying an "after burner" program to the final proton and neutron distributions. Spectators were not included in the calculations. It was shown that distributions are far from simple thermal Boltzmann distributions and the yield of light nuclei increases as one goes from midrapidity towards spectator rapidity regions. This effect was explained due to the strong correlation of nucleons in the RQMD source.

The total yields of ${ }^{3} \mathrm{He}$ have been estimated by fitting and integrating a parabolic parameterisation up to beam rapidity. These values shown in Fig. 4 (left) agree very well with a prediction of a statistical hadron gas model [12]. It is an overlooked aspect of the thermal model to compute the yields of composite particles reproduced with the chemical freeze-out parameters used to describe baryon and meson ratios [19]. In these investigations, an observed exponential decrease of composite particles implies a penalty factor for each edditional nucleon. In the relevant Boltzmann approximation this penalty factor, $R_{p} \approx \exp \frac{m \pm \mu_{b}}{T}$, where $m$ is a nucleon mass, can be easily derived. Using the chemical freeze-out parameters $\mu_{b}$ and $T$ appropriate for SPS energies obtained from the statistical model fit [20] to the NA49 data, the penalty factors were calculated as well as the yields of ${ }^{3} \mathrm{He}$. Fig. 4 (center) illustrates the results for the penalty factor $R_{p}$ as determined from $d n_{A} / d y=\mathrm{const} / R_{p}^{A-1}$ for AGS and SPS energies using the yields of $p, d$, and ${ }^{3} \mathrm{He}$. The values increase linearly with $\sqrt{s_{N N}}$ and are largest for $p_{t}=0$ yields. For $p_{t}$-integrated midrapidity yields $d n / d y$ the penalty factors decrease because of transverse flow. One finds their values to be 

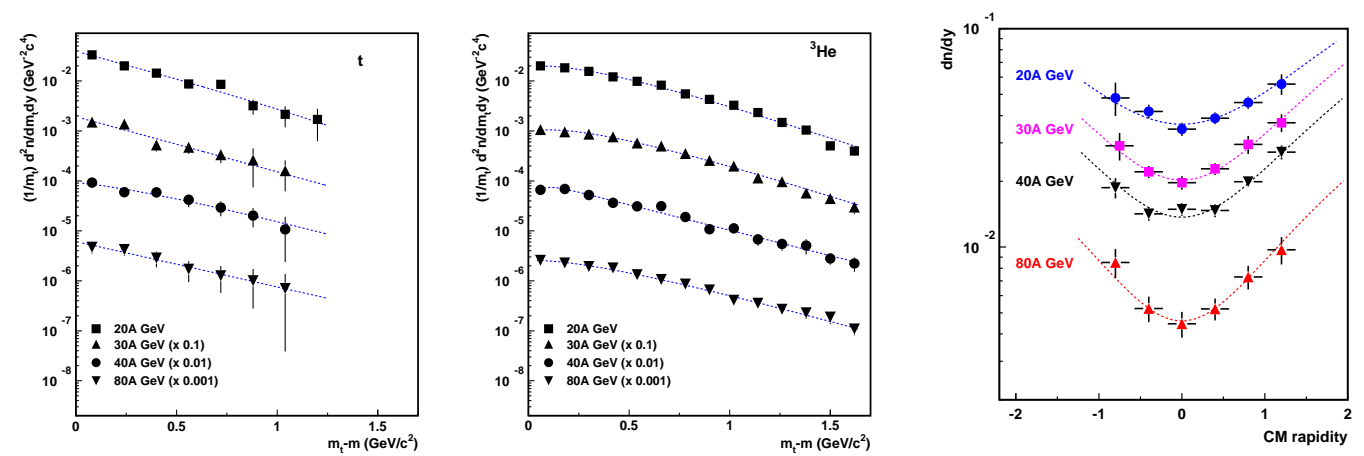

Figure 4: Transverse mass $m_{t}$ distributions of $t$ (left) and ${ }^{3} \mathrm{He}$ (center) at midrapidity in the $7 \%$ most central $\mathrm{Pb}+\mathrm{Pb}$ collisions at $20 \mathrm{~A}, 30 \mathrm{~A}, 40 \mathrm{~A}$ and $80 \mathrm{~A}$ GeVbeam energies. Rapidity distributions of ${ }^{3} \mathrm{He}$ (right). The dashed lines are fits with a parabola used to extract the total multiplicities.
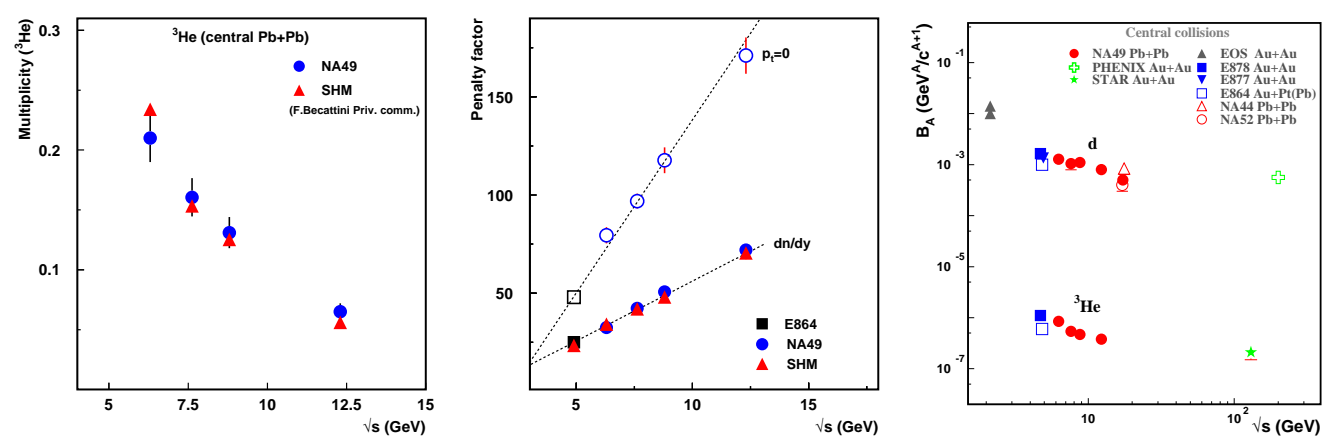

Figure 5: (left) Total multiplicity of ${ }^{3} \mathrm{He}$ in central $\mathrm{Pb}+\mathrm{Pb}$ collisions as a function of $\sqrt{s_{\mathrm{NN}}}$ (solid points). The triangles represent predictions of a statistical hadron gas model [12]. (center) Penalty factor derived from the midrapidity ${ }^{3} \mathrm{He}, d, p$ production yields in central $\mathrm{Pb}+\mathrm{Pb}$ collisions (dots) for $p_{t}=0$ and $p_{t}$-integrated yields compared to predictions of the statistical hadron gas model [12] (triangles). (right) Energy dependence of coalescence parameters $B_{2}$ and $B_{3}$ at $p_{t}=0$ for $d$ and ${ }^{3} \mathrm{He}$, respectively.

in surprising agreement with the statistical hadron gas model predictions [12]. As was mentioned above this agreement also holds for total ${ }^{3} \mathrm{He}$ yields. Both these observations might imply that ${ }^{3} \mathrm{He}$ is already formed at the chemical freeze-out, which might be in contradiction to the assumption of cluster formation via coalescence at possibly lower thermal freeze-out temperatures.

Using a general prescription of coalescence model which relates the invariant yield of light nuclei with mass $A$ to the $A$ th power of the proton yield [21], assuming the neuteron and proton distributions are identical:

$$
E_{A} \frac{d^{3} N_{A}}{d p_{A}^{3}}=B_{A}\left(E_{p} \frac{d^{3} N_{p}}{d p_{p}^{3}}\right)^{A}, p_{A}=A p_{p}
$$

the coalescence parameters $B_{2}$ and $B_{3}$ were extracted for deuterons and ${ }^{3} \mathrm{He}$, respectively. Their values at $p_{t}$ measured by NA49 are shown in Fig. 5 (right) and compared to results from lower 
and higher energies. One observes a gradual decrease with increasing beam energy which would suggest an increasing effective coalescence volume in the coalescence model scenario.

Analysis of antideuteron production was provided using 2.6 million events recorded in 158A $\mathrm{GeV} \mathrm{Pb}+\mathrm{Pb}$ collisions. For data set, a trigger requiring events from the $23 \%$ most central events was used by measuring the total energy of spectator nucleons and fragments in the veto calorimeter downstream of the target. Further off-line selection of the data for two classes of events corresponding to (0-10)\% and (10-23)\% centralities was used in analysis. The measurements were performed in rapidity range $2.0<y<2.5$ and transverse momentum $0<p_{t}<0.9 \mathrm{GeV} / c$. The yield of antideuterons as a function of $p_{t}$ is presented in Fig. 6 (left) together with that for deuterons scaled for comparison. A quite similar shapes of the transverse momentum distributions for $\bar{d}$ and $d$ in the common range of $p_{t}$ is observed from the figure. The ratio $\bar{d} / d \approx(2.0 \pm 0.2) \cdot 10^{-3}$ was calculated.

The coalescence parameter for antideuterons $B_{2}(\bar{d})$ for the $23 \%$ most central $\mathrm{Pb}+\mathrm{Pb}$ events as a function of $p_{t}$ is plotted in Fig. 6 (center). As seen from the data the $B_{2}(\bar{d})$ is almost independent on $p_{t}$ and its average value is calculated $B_{2}(\bar{d})=(9.1 \pm 0.9) \cdot 10^{-4} \mathrm{GeV}^{2} / c^{3}$. The coalescence parameters for $\bar{d}$ and $d$ as a function of the number of wounded nucleons for two selected centrality classes are shown in Fig. 6 (right). Being equal within errors $B_{2}$ for both $\bar{d}$ and $d$ increases with centrality of $158 \mathrm{~A} \mathrm{GeV} \mathrm{Pb}+\mathrm{Pb}$ collisions. This trend is usually attributed to the developed radial flow and strong transverse expansion of the system created in relativistic heavy ion rections.
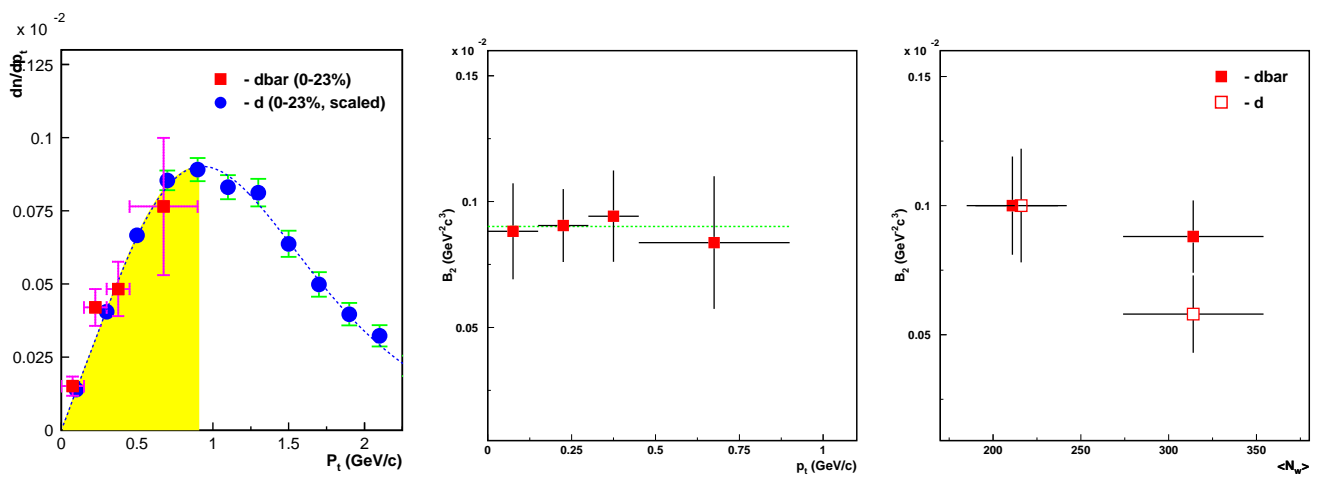

Figure 6: (left) Transverse momentum distributions for antideuterons $\left(0<p_{t}<0.9 \mathrm{GeV} / c^{2}\right)$ and that scaled for deuterons $\left.\left(0<p_{t}<2.0\right) \mathrm{GeV} / \mathrm{c}^{2}\right)$ in $158 \mathrm{~A} \mathrm{GeV} \mathrm{Pb}+\mathrm{Pb}$ collisions at $(0-23) \%$ centrality. Coalescence parameter $B_{2}$ for antideuterons as a function of $p_{t}$ (center) and the number of wounded nucleons $\left\langle N_{w}\right\rangle$ (right).

\section{Hyperon production}

The study of strange particle production in relativistic nucleus-nucleus collisions plays an important role because the strangeness enhancement is one of the primary signatures predicted for possible creation of the quark-gluon plasma.

New NA49 results on the $\Lambda$ and $\bar{\Lambda}$ production in minimum bias $\mathrm{Pb}+\mathrm{Pb}$ reactions at $40 A$ and $158 A$ GeVat midrapidity $\left(|y|<0.4\right.$ for $\Lambda(\bar{\Lambda})$ and $|y|<0.5$ for $\left.\Xi^{-}\right)$together with preliminary data on $\Xi^{-}[22]$ are presented. The midrapidity transverse mass distributions for these particles in each of the selected centality bins were obtained and the inverse slope parameters were calculated from the Boltzmann exponential fits to the data: 


$$
\frac{d^{2} n}{m_{t} d m_{t}}=C \cdot \exp ^{-\left(m_{t}-m\right) / T}
$$
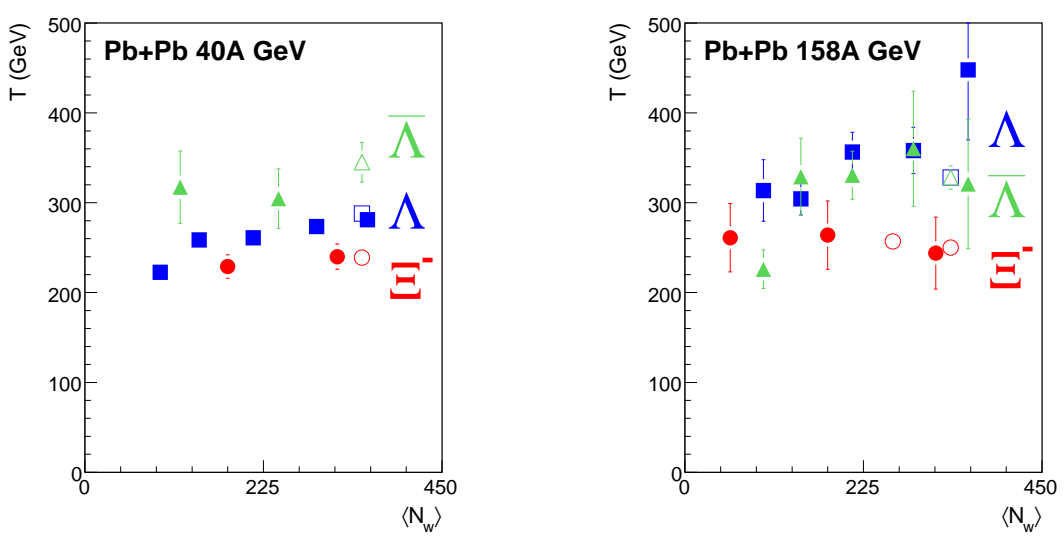

Figure 7: Inverse slope parameters for $\Lambda, \bar{\Lambda}$ and $\Xi^{-}$at midrapidity for minimum bias $\mathrm{Pb}+\mathrm{Pb}$ collisions (filled symbols) at $40 \mathrm{~A} \mathrm{GeV} \mathrm{(left)} \mathrm{and} 158 \mathrm{~A} \mathrm{GeV} \mathrm{(right)} \mathrm{as} \mathrm{a} \mathrm{function} \mathrm{of} \mathrm{the} \mathrm{number} \mathrm{of} \mathrm{wounded} \mathrm{nucleons}$ $\left\langle N_{w}\right\rangle$. Open symbols represents the results for online selected central reactions.

The centrality dependence of the inverse slope parameters for $40 \mathrm{~A}$ and $158 \mathrm{~A} \mathrm{GeV} \mathrm{Pb}+\mathrm{Pb}$ collisions is depicted in Fig. 7. It is determined in terms of the number of wounded nucleons $\left\langle N_{w}\right\rangle$ calculated within the Glauber model [23]. It is seen from the figures that the inverse slopes of $\Lambda$ and $\bar{\Lambda}$ slightly increase when going from peripheral to central $\mathrm{Pb}+\mathrm{Pb}$ collisions, whereas no centrality (system size) dependence is observed for $\Xi^{-}$cascade. The latter might be attributed to the early freeze-out of multistrange baryons $\Xi$ and $\Omega$ discussed in [24].

The centality dependence of hyperon yield (the rapidity density) per wounded nucleon for $\Lambda$, $\bar{\Lambda}$ and $\Xi^{-}$for $40 A$ and $158 A \mathrm{GeV}$ is shown in Fig. 8 (left and center). The yields were calculated from the measured transverse mass spectra and using a fitted function extrapolated to the full $m_{t}$ range. The $\Lambda$ data are corrected for feed down from weak decays. While there is no system size dependence of the rapidity densities per wounded nucleon for $\Lambda$ and $\bar{\Lambda}$, a weak rise can be observed in the case of the $\Xi^{-}$.

Having measured by NA49 the particle yields for $\mathrm{p}+\mathrm{p}$, central $\mathrm{C}+\mathrm{C}$ and $\mathrm{Si}+\mathrm{Si}$ [25], and minimum bias $\mathrm{Pb}+\mathrm{Pb}$ collisions at $158 \mathrm{~A} \mathrm{GeV}$ a hyperon enhancement factor $E$ can be determined as a ratio of the particle yields in $\mathrm{A}+\mathrm{A}$ to $\mathrm{p}+\mathrm{p}$ collisions:

$$
E=\left(\frac{d n / d y}{\left\langle N_{w}\right\rangle}\right)_{A A} /\left(\frac{d n / d y}{2}\right)_{p p}
$$

A behaviour of the enhancements with centrality of the collisions is displayed in Fig. 8 (right), where the data for selected centrality classes plotted as a function of the number of wounded nucleons. A clear hierarchy of the enhancement is visible. They increase with strangeness content of the particles $\left(E\left(\Xi^{-}\right)>E(\Lambda)>E(\bar{\Lambda})\right)$. For $\Xi^{-}$and $\Lambda$ a moderate $\left\langle N_{\mathrm{w}}\right\rangle$ dependence is seen while for the $\bar{\Lambda}$ it is compatible with being flat with centrality. About similar trends have been observed by the NA57 collaboration, however relative to a p+Be baseline [26]. The difference between 

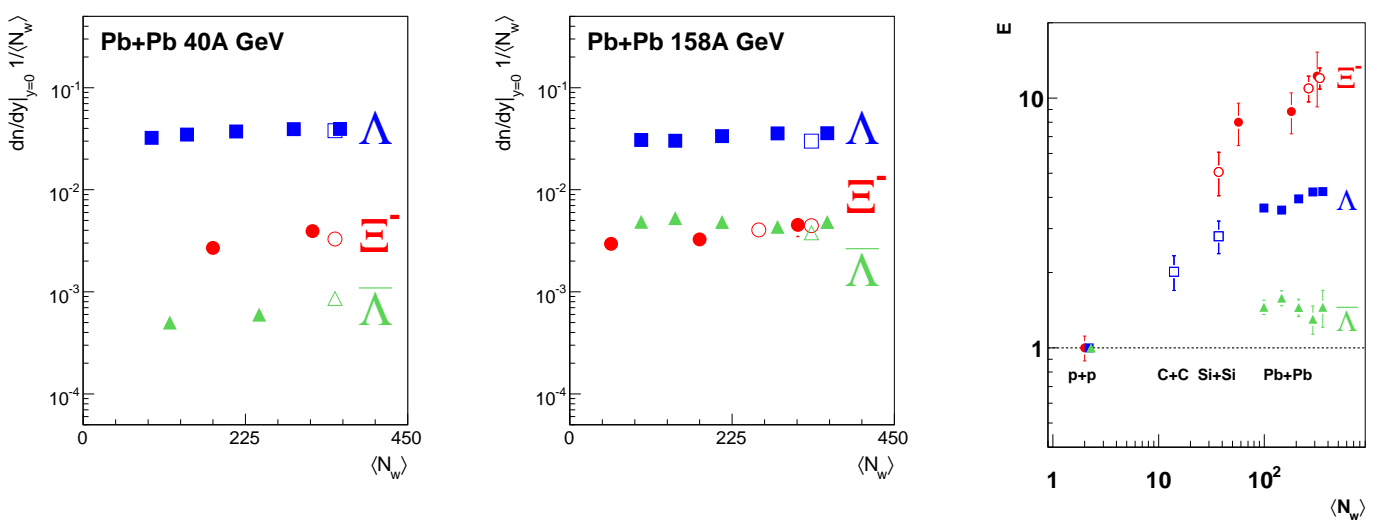

Figure 8: Rapidity densities per wounded nucleon of $\Lambda, \bar{\Lambda}$, and $\Xi^{-}$at midrapidity for minimum bias $\mathrm{Pb}+\mathrm{Pb}$ collisions (filled symbols) at $40 \mathrm{~A} \mathrm{GeV} \mathrm{(left)} \mathrm{and} 158 \mathrm{~A} \mathrm{GeV}$ (center) as a function of the number of wounded nucleons $\left\langle N_{\mathrm{w}}\right\rangle$. Open symbols represent the results for online selected central reactions. (right) The midrapidity yields per wounded nucleon relative to $\mathrm{p}+\mathrm{p}$ yields for central $\mathrm{C}+\mathrm{C}, \mathrm{Si}+\mathrm{Si}$ and minimum bias $\mathrm{Pb}+\mathrm{Pb}$ reactions at $158 \mathrm{~A} \mathrm{GeVas}$ a function of $\left\langle N_{\mathrm{w}}\right\rangle$.

$\Lambda$ and $\bar{\Lambda}$ is unclear. This could be due to a difference in the initial production mechanism or a centrality-dependent $\bar{\Lambda}$ absorption in a baryon-rich environment.

\section{Conclusions}

New results were presented on the energy dependence of baryon stopping, production of light (anti)nuclei ${ }^{3} \mathrm{He}, t, \bar{d}$ and $d$ and centrality dependence of (anti)strange baryons $\Lambda, \bar{\Lambda}$, and $\Xi^{-}$in $\mathrm{Pb}+\mathrm{Pb}$ collisions throughout the CERN SPS energy range.

A clear evolution with energy from a peak to a dip structure is seen in the net baryon distributions within $20 A-158 A \mathrm{GeV}$ energy range. The relative net baryon rapidity shift $\langle\delta y\rangle / y_{\text {proj }} \approx 0.6$ at the AGS and SPS and slowly decreases towards RHIC energies. The estimated inelasticity coefficient indicate that average energy loss of projectiles amounts to about (70-80)\%.

The quantitative results on the energy dependence of total multiplicity of ${ }^{3} \mathrm{He}$ and penalty factor derived from ${ }^{3} \mathrm{He}, d$ and $p$ data are in remarkable agreement with statistical hadron gas model predictions. The measured yields of the light nuclei $\bar{d}$ and $d$ together with those for ${ }^{-} p$ and $p$ at midrapidity and nonzero $p_{t}$ in the $23 \%$ most central $\mathrm{Pb}+\mathrm{Pb}$ collisions at $158 \mathrm{~A} \mathrm{GeV}$ has allowed to extract and compare a coalescence parameters $B_{2}(\bar{d})$ and $B_{2}(d)$. Their values turned out to be almost similar with no visible dependence on the transverse momentum as expected from the coalescence models.

The hyperon yields per wounded nucleon at $158 \mathrm{~A} \mathrm{GeV} \mathrm{Pb}+\mathrm{Pb}$ collisions are enhanced with respect to $p+p$ interactions. A visible centrality dependence of the strangeness enhancement is observed for $\Lambda$ and $\Xi^{-}$and not for $\bar{\Lambda}$ hyperons. The inverse slope chracteristics of the measured hyperon $m_{t}$ distributions at various centralities could support the conception of early freeze-out of multistrange hyperons. 


\section{Acknowledgments}

Sincere thanks to the organisers for a stimulating workshop and for the opportunity to show results from NA49.

This work was supported by the US Department of Energy Grant DE-FG03-97ER41020/A000, the Bundesministerium fur Bildung und Forschung, Germany, the Virtual Institute VI-146 of Helmholtz Gemeinschaft, Germany, the Polish State Committee for Scientific Research (1 P03B 006 30, 1 P03B 097 29 , 1 PO3B 121 29, 1 P03B 127 30), the Hungarian Scientific Research Foundation (T032648, T032293, T043514), the Hungarian National Science Foundation, OTKA, (F034707), the Polish-German Foundation, the Korea Science \& Engineering Foundation (R01-2 005-00010334-0), the Bulgarian National Science Fund (Ph-09/05) and the Croatian Ministry of Science, Education and Sport (Project 098-0982887-2878).

\section{References}

[1] M. Gazdzicki, Report from NA49, J.Phys. G30, S701 (2004).

[2] P. Seyboth, Onset of deconfinement in Pb+Pb collisions at the CERN SPS, Acta Phys. Pol B37, 3429 (2006).

[3] S. Afanasiev et al., The NA49 large acceptance hadron detector, Nucl. Instrum. Meth. A430, 210 (1999).

[4] S. Afanasiev et al., Energy dependence of pion and kaon production in central $\mathrm{Pb}+\mathrm{Pb}$ collisions Phys. Rev. C 66 (2002) 054902.

[5] C. Alt et al., Energy and centrality dependence of $\bar{p}$ and $p$ production and the $\bar{\Lambda} \bar{p}$ ratio in $\mathrm{Pb}+\mathrm{Pb}$ collisions between 20A GeV and 158A GeV, Phys. Rev. C73, 044910 (2006).

[6] T. Anticic et al., $\Lambda$ and $\bar{\Lambda}$ production in central $P b+P b$ collisions at 40,80 and $158 \mathrm{~A} \mathrm{GeV}$, Phys. Rev. Lett. 93, 022302 (2004).

[7] C. Alt et al., $\Omega$ and $\bar{\Omega}$ production in central $P b+P b$ collisions at $40 \mathrm{~A} \mathrm{GeV}$ and $158 \mathrm{~A} \mathrm{GeV \text {, }}$ Phys. Rev. Lett. 94, 192301 (2005).

[8] H. Appelshäuser, Baryon Stopping and Charged Particle Distributions in Central Pb+Pb Collisions at $158 \mathrm{GeV/c}$ per Nucleon, Phys.Rev.Lett. 82, 2471 (1999).

[9] L. Ahle et al., Proton and deuteron production in Au+Au reactions at 11.6GeV/c, Phys. Rev. C60, 064901 (1999).

[10] I. G. Bearden et al., Nuclear stopping in Au+Au collisions at $\sqrt{s_{N N}}=200 \mathrm{GeV}$, Phys. Rev. Lett. 93, 102301 (2004).

[11] H. Weber et al., Nucleus-Nucleus collisions at high baryon densities, Phys. Lett. B545, 285 (2002).

[12] F. Becattini et al., Energy and system size dependence of chemical freeze-out in relativistic nuclear collisions, Phys. Rev. C73, 044905 (2006) and private communication.

[13] A. Polleri et al., Effect of collective expansion on light cluster spectra in relativistic heavy ion collisions, Phys. Lett. B419, 19 (1998).

[14] R. Scheibl and U. Heinz, Coalescence and flow in ultrarelativistic heavy ion collisions, Phys. Rev. C59, 1585 (1999). 
[15] T. Anticic et al., Energy and centrality dependence of deuteron and proton production in $\mathrm{Pb}+\mathrm{Pb}$ collisions at relativistic energies, Phys. Rev. C69, 024902 (2004).

[16] R. Mattiello et al., Nuclear clusters as a probe for expansion flow in heavy ion reactions at (10-15)A GeV, Phys. Rev. C55, 1443 (1997).

[17] A. Hansen, Light nuclei production in ultrarelativistic heavy ion collisions, PhD thesis, The Niels Bohr Institute. (August 1999).

[18] M. J. Bennett et al., Light nuclei production in relativistic Au+nucleus collisions, Phys. Rev. C58, 1155 (1998).

[19] P. Braun-Munzinger and J. Stachel, Particle ratios, equilibration and the QCD phase boundary, J.Phys. G28, 1971-1976 (2002).

[20] F. Becattini et al., Chemical equilibrium study in nucleus-nucleus collisions at relativistic energies, Phys. Rev. C69, 024905 (2004).

[21] V. J. Llope et al., The fragment coalescence model, Phys. Rev. C52, 2004 (1995).

[22] M. Mitrovski et al., Strangeness production at SPS energies, J. Phys. G32, S43 (2006).

[23] R. J. Glauber and G. Matthiae et al., High-energy scattering of protons by nuclei, Nucl. Phys. B21, 135 (1970).

[24] H. van Hecke. et al., Evidence of early multi-strange hadron freeze-out in high energy nuclear collisions, Nucl.Phys. A661 (1999) 493c-496c.

[25] C. Alt et al., System-size dependence od strangeness production in nucleus-nucleus collisions at $\sqrt{s_{N N}} / 2=17.3 \mathrm{GeV}$, Phys. Rev. Lett. 94, 052301 (2005).

[26] F. Antinori, Enhancement of hyperon production at central rapidity in $158 \mathrm{~A} \mathrm{CeV/c} \mathrm{Pb}-\mathrm{Pb}$ collisions, J.Phys. G32, 427 (2006). 